\title{
Article \\ Dynamic Event-Triggered Adaptive Tracking Control for a Class of Unknown Stochastic Nonlinear Strict-Feedback Systems
}

\author{
Yingying $\mathrm{Fu}^{1}$, Jing $\mathrm{Li}^{1, *(\mathbb{D})}$, Shuiyan $\mathrm{Wu}^{2}$ and Xiaobo $\mathrm{Li}^{3}$ \\ 1 School of Mathematics and Statistics, Xidian University, Xi'an 710071, China; yyfu@stu.xidian.edu.cn \\ 2 College of Mathematics and Information Science, Xianyang Normal University, Xianyang 712099, China; \\ shuiyanwu@xync.edu.cn \\ 3 School of Mathematics and Information Science, Baoji University of Arts and Sciences, Baoji 721013, China; \\ xlyang_0108@stu.xidian.edu.cn \\ * Correspondence: lijingmath@xidian.edu.cn; Tel.: +86-136-791-78646
}

Citation: Fu, Y.; Li, J.; Wu, S.; Li, X. Dynamic Event-Triggered Adaptive Tracking Control for a Class of Unknown Stochastic Nonlinear Strict-Feedback Systems. Symmetry 2021, 13, 1648. https://doi.org/ $10.3390 /$ sym 13091648

Academic Editors: Aviv Gibali and Calogero Vetro

Received: 23 July 2021

Accepted: 4 September 2021

Published: 7 September 2021

Publisher's Note: MDPI stays neutral with regard to jurisdictional claims in published maps and institutional affiliations.

Copyright: (c) 2021 by the authors. Licensee MDPI, Basel, Switzerland. This article is an open access article distributed under the terms and conditions of the Creative Commons Attribution (CC BY) license (https:/ / creativecommons.org/licenses/by/ $4.0 /)$.

\begin{abstract}
In this paper, the dynamic event-triggered tracking control issue is studied for a class of unknown stochastic nonlinear systems with strict-feedback form. At first, neural networks (NNs) are used to approximate the unknown nonlinear functions. Then, a dynamic event-triggered controller (DETC) is designed through the adaptive backstepping method. Especially, the triggered threshold is dynamically adjusted. Compared with its corresponding static event-triggered mechanism (SETM), the dynamic event-triggered mechanism (DETM) can generate a larger execution interval and further save resources. Moreover, it is verified by two simulation examples that show that the closed-loop stochastic system signals are ultimately fourth moment semi-globally uniformly bounded (SGUUB).
\end{abstract}

Keywords: stochastic nonlinear system; dynamic event-triggered controller; neural network; adaptive backstepping

\section{Introduction}

In actual engineering applications, network bandwidth is usually limited, and there exist multiple devices sharing the same network. Therefore, we must consider the utilization rate of communication resources. One of the major solutions is to reduce the amount of data transmission as much as possible while ensuring the overall performance of the system. Traditional time-triggered mechanisms can reduce the times of communication to some extent, but it is not flexible enough from the perspective of resource utilization. Compared with time-triggered mechanisms, an event-triggered mechanism (ETM) can reduce the amount of data transmission more effectively, decrease the network load, and have higher flexibility. Thus, scholars have turned their attention to the study of ETM [1-14]. In [2], the authors illustrate the advantages of ETM in saving resources through experimental methods. For uncertain nonlinear systems [5,6], Xing et al. put forward three different ETMs through the adaptive backstepping design method. In [11,12], the authors consider the tracking control problem of stochastic nonlinear systems with an uncertain term. Two adaptive event-triggered controllers with fixed threshold and relative threshold are designed based on fuzzy logic system.

However, most of the above-referred works on ETM are inspired by the work in [3], and the threshold of ETM is commonly a fixed value, so this ETM can be interpreted as SETM. Based on the framework of [3], Girard proposes DETM for the first time in [15], which introduces an intermediate dynamic variable in the triggering condition for adaptive adjustment of the threshold value. In comparison with SETM, DETM can generate a larger execution interval and save resources more effectively. Therefore, DETM is used to solve different problems [15-28]. For networked switched linear systems with time-varying delays [18], a DETM is constructed to guarantee the exponential stability of the closed-loop system, and the simulation comparison is performed to show that the proposed DETM can 
reduce the times of data transmissions. Considering the consensus problem of linear multiagent systems [22], DETM is added into the design of the proposed distributed control protocol. Furthermore, for the optimal control problem of locally unknown nonlinear systems, an integral reinforcement learning algorithm based on DETM is proposed in [26], and it is demonstrated that DETM can obtain a larger average triggered interval than SETM under similar performance. When the system is subject to external disturbances and sensor saturation [27], Zuo et al. propose an output-feedback control scheme with DETM to solve the stabilization problem. In [28], the tracking control issue is investigated for unknown nonlinear systems, and the authors designed an adaptive DETC by combing with NN.

Obviously, most of DETM investigations mentioned above are currently focused on deterministic systems. In addition, the systems with environmental disturbances and uncertainty components are often modeled as stochastic nonlinear systems [29,30]. Relative to deterministic systems, the stochastic nonlinear system model can more accurately reflect the dynamic features of the real system. Therefore, it is very significant to study stochastic nonlinear systems. In this paper, for a class of unknown stochastic nonlinear strict-feedback systems, an adaptive DETC is designed in combination with NNs [31-39]. The designed DETC ensures that all the signals in the closed-loop system are SGUUB in the sense of the fourth moment.

The main contributions of this paper are summarized below:

(1) A class of unknown stochastic nonlinear strict-feedback systems are considered in this paper, which differ from the deterministic systems studied in [28]. Obviously, stochastic nonlinear systems can reflect the dynamic features of real systems more accurately and are more general. In addition, the unknown nonlinear functions in this system are approximated by NNs.

(2) An adaptive controller with DETM is designed for unknown stochastic nonlinear strict-feedback systems. Compared with SETM designed in [11,12], DETM can save more resources.

The remainder of this paper is arranged as follows. In Section 2, we provide some basic knowledge and problem formulation. In Section 3, we give a description of the design process of the adaptive controller with DETM. Section 4 conducts the simulation. Finally, the conclusions are drawn in Section 5.

Notation 1. The notations of this paper is explained as follows: $\mathbb{R}^{n \times r}, \mathbb{R}^{n}$, and $\mathbb{R}$ represent the sets of real $n \times r$ matrix space, real $n$-dimensional vector space, and real numbers, respectively. For vector $g$, its transpose can be depicted as $g^{T}$. A function $\mathcal{K}:[0, \infty) \rightarrow[0, \infty)$ is of class $\mathcal{K}_{\infty}$, if $\mathcal{K}$ is continuous, strictly increasing and $\mathcal{K}(0)=0, \lim _{r \rightarrow \infty} \mathcal{K}(r)=\infty$. The expectation operator is expressed as $E(\cdot) . C^{i}$ represents the set of all functions with continuous ith partial derivatives; $C^{2,1}$ denotes the family of all non-negative functions $V(x, t)$, which are $C^{2}$ in $x$ and $C^{1}$ in $t . \operatorname{Tr}(\cdot)$ is the trace of a matrix. $Z^{+}$denotes the set of positive integers.

\section{Problem Statement and Preliminary}

\subsection{Problem Statement}

Consider the following stochastic nonlinear strict-feedback system

$$
\left\{\begin{array}{l}
d x_{j}=\left[x_{j+1}+\varphi_{j}\left(\bar{x}_{j}\right)\right] d t+g_{j}^{T}\left(\bar{x}_{j}\right) d w, j=1, \ldots, n-1, \\
d x_{n}=\left[u(t)+\varphi_{n}\left(\bar{x}_{n}\right)\right] d t+g_{n}^{T}\left(\bar{x}_{n}\right) d w \\
y=x_{1}
\end{array}\right.
$$

where $\bar{x}_{n}=\left[x_{1}, \ldots, x_{n}\right]^{T} \in \mathbb{R}^{n}$ represents the system state vector; $u(t) \in \mathbb{R}$ and $y \in \mathbb{R}$ are control input and system output, respectively; $\varphi_{j}\left(\bar{x}_{j}\right): \mathbb{R} \rightarrow \mathbb{R}^{n}$ and $g_{j}\left(\bar{x}_{j}\right): \mathbb{R} \rightarrow \mathbb{R}^{r}$ are unknown smooth nonlinear functions satisfying $\varphi_{j}(0)=g_{j}(0)=0 ; w$ is $r$-dimensional independent standard Wiener process. For simplicity, we denote $\varphi_{j}\left(\bar{x}_{j}\right)$ as $\varphi_{j}$ and $g_{j}\left(\bar{x}_{j}\right)$ as $g_{j}$. 
Our control objective is to design an adaptive DETC $u$ so that the system output $y$ can track the reference signal $y_{d}$, and all the signals of the closed-loop system are SGUUB in the sense of the fourth moment.

To obtain this target, some related definitions, assumptions, and lemmas are introduced next, which are of great importance for the design process below. Firstly, consider the stochastic nonlinear system:

$$
d x=f(x) d t+g(x) d w
$$

where $f(x) \in \mathbb{R}^{n}$ and $g(x) \in \mathbb{R}^{n \times r}$ are locally Lipschitz functions, which satisfy $f(0)=0$ and $g(0)=0$.

Assumption 1. (see, [11]). The desired trajectory $y_{d}(t)$ and its first $n$th order derivatives are known, continuous, and bounded.

Definition 1. (see, [11]). For $\forall V(x) \in C^{2}$, the infinitesimal generator $\mathcal{L}$ is defined as

$$
\mathcal{L} V=\frac{\partial V}{\partial x} f+\frac{1}{2} \operatorname{Tr}\left\{g^{T} \frac{\partial^{2} V}{\partial x^{2}} g\right\}
$$

Definition 2. (see, [11]). If for a compact set $\Omega \in \mathbb{R}$ and any initial state $x\left(t_{0}\right)=x_{0} \in \Omega$, there exist a constant $\epsilon>0$ and a time constant $T=T\left(\epsilon, x_{0}\right)$ such that $E\left(\| x\left(t \|^{b}\right)<\epsilon\right.$ for all $t>t_{0}+T$, the trajectory $\{x(t), t \geq 0\}$ of system (2) is called to be SGUUB in the bth moment.

Lemma 1. (see, [11]). For system (2), if there exist functions $a_{1}, a_{2} \in \mathcal{K}_{\infty}$, positive constants $N$, $\Lambda$, and function $V(x, t) \in C^{2,1}$ to satisfy

$$
\left\{\begin{array}{l}
a_{1}(\|x\|) \leq V(x, t) \leq a_{2}(\|x\|) \\
\mathcal{L} V \leq-N V+\Lambda
\end{array}\right.
$$

then system (2) has a unique solution and satisfies

$$
E[V(x, t)] \leq V\left(x_{0}\right) e^{-N t}+\frac{\Lambda}{N}, \forall t>t_{0}
$$

Lemma 2 (Young's Inequality). (see, [11]). If constants $a, l_{1}, l_{2}>0$ and $\left(l_{1}-1\right)$ $+\left(l_{2}-1\right)=1$, then

$$
p^{T} q \leq \frac{a^{l_{1}}}{l_{1}}\|p\|^{l_{1}}+\frac{1}{l_{2} a_{2}}\|q\|^{l_{2}}, \forall p, q \in \mathbb{R}^{n} .
$$

\subsection{Function Approximation}

In the following, the radial basis function NNs [36] are employed to settle unknown nonlinear continuous functions, which means that the continuous function $A_{j}$ can be approximated on a compact set $D$ as:

$$
A_{j}=W_{j}^{T} S_{j}\left(X_{j}\right)+\xi_{j}\left(X_{j}\right)
$$

where $W_{j} \in \mathbb{R}^{l}$ and $\xi_{j}\left(X_{j}\right) \in \mathbb{R}$ are NN ideal weights and approximation error, respectively; The NN node number is $l ; X_{j}=\left[x_{1}, x_{2}, \ldots, x_{j}\right]^{T}(1 \leq j \leq n)$ is the NN input vector; $S_{j}=\left[S_{j}^{1}, S_{j}^{2}, \ldots, S_{j}^{l}\right]^{T}: D \rightarrow \mathbb{R}^{l}$ is the radial basis function and can be selected in the form of the following Gaussian function

$$
S_{j}=\exp \left[-\frac{\left\|x_{j}-\mu_{j}\right\|^{2}}{2 \sigma_{j}^{2}}\right] ;
$$


$\sigma_{j}>0$ and $\mu_{j} \in D$ are the width and center of basis function $S_{j}$, respectively; Define the ideal $W_{j}$ as

$$
W_{j}=\arg \min _{\hat{W}_{j} \in \mathbb{R}^{l}}\left\{\sup _{X_{j} \in D}\left|A_{j}-\hat{W}_{j}^{T} S_{j}\left(X_{j}\right)\right|\right\} .
$$

Now, we give the following assumption about the approximation error $\xi_{j}\left(X_{j}\right)$ :

Assumption 2. (see, [36]). The approximation error $\xi_{j}\left(X_{j}\right)(j=1,2, \ldots, n)$ satisfies

$$
\left\|\xi_{j}\left(X_{j}\right)\right\| \leq \delta_{j}
$$

where $\delta_{j}>0$ is a constant.

\section{DETC Design and Stability Analysis}

To design an adaptive DETC, we define the following coordinate transformation:

$$
\left\{\begin{array}{l}
z_{1}=x_{1}-y_{d}, \\
z_{j}=x_{j}-\alpha_{j-1}, j=2, \ldots, n,
\end{array}\right.
$$

where $z_{j}$ represents the error variable, $\alpha_{j-1}$ is the virtual controller, which need to be designed subsequently.

Now, the adaptive backstepping method is used for the following design.

Step 1.

$$
\begin{aligned}
d z_{1} & =d\left(x_{1}-y_{d}\right) \\
& =\left(x_{2}+\varphi_{1}-\dot{y}_{d}\right) d t+g_{1}^{T} d w
\end{aligned}
$$

Select a symmetric positive-definite Lyapunov function

$$
V_{0}=\frac{1}{4} z_{1}^{4}
$$

There holds

$$
\begin{aligned}
\mathcal{L} V_{0} & =z_{1}^{3}\left(x_{2}+\varphi_{1}-\dot{y}_{d}\right)+\frac{3}{2} z_{1}^{2} g_{1}^{T} g_{1} \\
& =z_{1}^{3}\left(z_{2}+\alpha_{1}+\varphi_{1}-\dot{y}_{d}\right)+\frac{3}{2} z_{1}^{2} g_{1}^{T} g_{1} .
\end{aligned}
$$

with Young's Inequality, we acquire

$$
z_{1}^{3} z_{2} \leq \frac{3}{4} z_{1}^{4}+\frac{1}{4} z_{2}^{4}
$$

and

$$
\frac{3}{2} z_{1}^{2} g_{1}^{T} g_{1} \leq \frac{9}{8} z_{1}^{4}\left\|g_{1}\right\|^{4}+\frac{1}{2} .
$$

Putting (6) and (7) into (5), formula (5) can be expressed as

$$
\mathcal{L} V_{0} \leq z_{1}^{3}\left(\frac{3}{4} z_{1}+\alpha_{1}+A_{1}-\dot{y}_{d}\right)+\frac{1}{4} z_{2}^{4}+\frac{1}{2}
$$

where $A_{1}=\varphi_{1}+\frac{9}{8} z_{1}\left\|g_{1}\right\|^{4}$.

Since functions $\varphi_{1}$ and $g_{1}$ are unknown, function $A_{1}$ can be approximated as $A_{1}=W_{1}^{T} S_{1}\left(X_{1}\right)+\xi_{1}\left(X_{1}\right)$ through using $\mathrm{NN}$, where $\xi_{1}\left(X_{1}\right)$ is the $\mathrm{NN}$ approximation error, $\left\|\xi_{1}\left(X_{1}\right)\right\| \leq \delta_{1}$, and $\delta_{1}>0$. 
Using Young's Inequality, we get

$$
\begin{aligned}
z_{1}^{3} A_{1} & =z_{1}^{3} W_{1}^{T} S_{1}\left(X_{1}\right)+z_{1}^{3} \xi_{1}\left(X_{1}\right) \\
& \leq \frac{z_{1}^{6} S_{1}^{T} S_{1}}{2 c_{1}^{2}} \theta+\frac{c_{1}^{2}}{2}+\frac{z_{1}^{6}}{2}+\frac{\delta_{1}^{2}}{2}
\end{aligned}
$$

where $c_{1}>0$, and $\theta=\max _{1 \leq j \leq n}\left\{\left\|W_{j}\right\|^{2}\right\}$.

Remark 1. To reduce the number of adjustments of the adaptive parameters, we redefine a new unknown parameter $\theta$ in this paper, so that only one adaptive parameter needs to be estimated online during the whole design process. The remaining $N N$ weight $W_{j}$ will appear in the subsequent design process. For convenience, the lumped parameter is expressed as $\theta=\max _{1 \leq j \leq n}\left\{\left\|W_{j}\right\|^{2}\right\}$.

Choose

$$
V_{1}=V_{0}+\frac{1}{2 \gamma} \tilde{\theta}^{2}
$$

where $\tilde{\theta}=\theta-\hat{\theta}$ is estimation error of $\theta$.

Moreover, we have

$$
\mathcal{L} V_{1}=\mathcal{L} V_{0}-\frac{1}{\gamma} \tilde{\theta} \dot{\hat{\theta}}
$$

Substituting (8) and (9) into the above equation, we obtain

$$
\begin{aligned}
\mathcal{L} V_{1} \leq & z_{1}^{3}\left(\frac{3}{4} z_{1}+\alpha_{1}+\frac{z_{1}^{3} S_{1}^{T} S_{1}}{2 c_{1}^{2}} \hat{\theta}+\frac{z_{1}^{3}}{2}-\dot{y}_{d}\right)+\frac{1}{\gamma} \tilde{\theta}\left(\frac{\gamma z_{1}^{6} S_{1}^{T} S_{1}}{2 c_{1}^{2}}-\dot{\hat{\theta}}\right) \\
& +\frac{1}{4} z_{2}^{4}+\frac{1}{2}+\frac{\left(c_{1}^{2}+\delta_{1}^{2}\right)}{2} .
\end{aligned}
$$

To reach our control objective, we choose virtual control variable $\alpha_{1}$ as

$$
\alpha_{1}=-\left(k_{1}+\frac{3}{4}\right) z_{1}-\frac{z_{1}^{3} S_{1}^{T} S_{1}}{2 c_{1}^{2}} \hat{\theta}-\frac{z_{1}^{3}}{2}+\dot{y}_{d}
$$

where $k_{1}>0$ is a constant and

$$
\varsigma_{1}=\frac{\gamma z_{1}^{6} S_{1}^{T} S_{1}}{2 c_{1}^{2}}
$$

Substituting (11) and (12) into (10), we obtain

$$
\mathcal{L} V_{1} \leq-k_{1} z_{1}^{4}+\frac{1}{\gamma} \tilde{\theta}\left(\varsigma_{1}-\dot{\hat{\theta}}\right)+\frac{1}{4} z_{2}^{4}+\frac{1}{2}+\frac{\left(c_{1}^{2}+\delta_{1}^{2}\right)}{2} .
$$

Step $j(2 \leq j \leq n-1)$.

$$
\begin{aligned}
d z_{j}= & d x_{j}-d \alpha_{j-1} \\
= & \left(x_{j+1}+\varphi_{j}\right) d t+g_{j}^{T} d w-d \alpha_{j-1} \\
= & \left(x_{j+1}+\varphi_{j}-\sum_{i=1}^{j-1} \frac{\partial \alpha_{j-1}}{\partial x_{i}} \varphi_{i}-\frac{1}{2} \sum_{i, m=1}^{j-1} \frac{\partial^{2} \alpha_{j-1}}{\partial x_{i} \partial x_{m}} g_{i}^{T} g_{m}-B_{j}\right) d t \\
& +\left(g_{j}^{T}-\sum_{i=1}^{j-1} \frac{\partial \alpha_{j-1}}{\partial x_{i}} g_{i}^{T}\right) d w,
\end{aligned}
$$

where $B_{j}=\frac{\partial \alpha_{j-1}}{\partial \hat{\theta}} \dot{\hat{\theta}}+\sum_{i=1}^{j-1} \frac{\partial \alpha_{j-1}}{\partial x_{i}} x_{i+1}+\sum_{i=0}^{j-1} \frac{\partial \alpha_{j-1}}{\partial y_{d}^{(i)}} y_{d}^{(i+1)}$. 
Select the following symmetric positive-definite Lyapunov function

$$
V_{j}=V_{j-1}+\frac{1}{4} z_{j}^{4}
$$

There holds

$$
\begin{aligned}
\mathcal{L} V_{j}= & \mathcal{L} V_{j-1}+z_{j}^{3}\left(x_{j+1}+\varphi_{j}-\sum_{i=1}^{j-1} \frac{\partial \alpha_{j-1}}{\partial x_{i}} \varphi_{i}-\frac{1}{2} \sum_{i, m=1}^{j-1} \frac{\partial^{2} \alpha_{j-1}}{\partial x_{i} \partial x_{m}} g_{i}^{T} g_{m}-B_{j}\right) \\
& +\frac{3}{2} z_{j}^{2}\left(g_{j}^{T}-\sum_{i=1}^{j-1} \frac{\partial \alpha_{j-1}}{\partial x_{i}} g_{i}^{T}\right)^{2} \\
= & \mathcal{L} V_{j-1}+z_{j}^{3}\left(z_{j+1}+\alpha_{j}+\varphi_{j}-\sum_{i=1}^{j-1} \frac{\partial \alpha_{j-1}}{\partial x_{i}} \varphi_{i}-\frac{1}{2} \sum_{i, m=1}^{j-1} \frac{\partial^{2} \alpha_{j-1}}{\partial x_{i} \partial x_{m}} g_{i}^{T} g_{m}\right. \\
& \left.-B_{j}\right)+\frac{3}{2} z_{j}^{2}\left(g_{j}^{T}-\sum_{i=1}^{j-1} \frac{\partial \alpha_{j-1}}{\partial x_{i}} g_{i}^{T}\right)^{2} .
\end{aligned}
$$

Applying Young's inequality, we have

$$
\begin{aligned}
z_{j}^{3} z_{j+1} & \leq \frac{3}{4} z_{j}^{4}+\frac{1}{4} z_{j+1}^{4} \\
\frac{3}{2} z_{j}^{2}\left(g_{j}^{T}-\sum_{i=1}^{j-1} \frac{\partial \alpha_{j-1}}{\partial x_{i}} g_{i}^{T}\right)^{2} & \leq \frac{9}{8} z_{j}^{4}\left(g_{j}^{T}-\sum_{i=1}^{j-1} \frac{\partial \alpha_{j-1}}{\partial x_{i}} g_{i}^{T}\right)^{4}+\frac{1}{2} .
\end{aligned}
$$

Putting (15) and (16) into (14), we further get

$$
\mathcal{L} V_{j} \leq \mathcal{L} V_{j-1}+z_{j}^{3}\left(\frac{3}{4} z_{j}+\alpha_{j}+A_{j}-B_{j}\right)+\frac{1}{4} z_{j+1}^{4}+\frac{1}{2},
$$

where $A_{j}=\varphi_{j}-\sum_{i=1}^{j-1} \frac{\partial \alpha_{j-1}}{\partial x_{i}} \varphi_{i}-\frac{1}{2} \sum_{i, m=1}^{j-1} \frac{\partial^{2} \alpha_{j-1}}{\partial x_{i} \partial x_{m}} g_{i}^{T} g_{m}+\frac{9}{8} z_{j}\left(g_{j}^{T}-\sum_{i=1}^{j-1} \frac{\partial \alpha_{j-1}}{\partial x_{i}} g_{i}^{T}\right)^{4}$.

Function $A_{j}$ can be approximated as $A_{j}=W_{j}^{T} S_{j}\left(X_{j}\right)+\xi_{j}\left(X_{j}\right)$ by using $\mathrm{NN}$, where $\xi_{j}\left(X_{j}\right)$ is the $\mathrm{NN}$ approximation error, $\left\|\xi_{j}\left(X_{j}\right)\right\| \leq \delta_{j}$, and $\delta_{j}>0$. Using Young's Inequality, we get

$$
\begin{aligned}
z_{j}^{3} A_{j} & =z_{j}^{3} W_{j}^{T} S_{j}+z_{j}^{3} \xi_{i} \\
& \leq \frac{z_{j}^{6} S_{j}^{T} S_{j}}{2 c_{j}^{2}} \theta+\frac{c_{j}^{2}}{2}+\frac{z_{j}^{6}}{2}+\frac{\delta_{j}^{2}}{2}
\end{aligned}
$$

where $c_{j}>0$.

Combining (17) and (18), the following inequality is derived

$$
\begin{aligned}
\mathcal{L} V_{j} \leq & \mathcal{L} V_{j-1}+z_{j}^{3}\left(\frac{3}{4} z_{j}+\alpha_{j}+\frac{z_{j}^{3} S_{j}^{T} S_{j}}{2 c_{j}^{2}} \theta+\frac{z_{j}^{3}}{2}-B_{j}\right)+\frac{1}{4} z_{j+1}^{4} \\
& +\frac{1}{2}+\frac{\left(c_{j}^{2}+\delta_{j}^{2}\right)}{2} \\
\leq & -\sum_{i=1}^{j-1} k_{i} z_{i}^{4}+z_{j}^{3}\left(z_{j}+\alpha_{j}+\frac{z_{j}^{3} S_{j}^{T} S_{j}}{2 c_{j}^{2}} \hat{\theta}+\frac{z_{j}^{3}}{2}-B_{j}\right)+\frac{1}{4} z_{j+1}^{4} \\
& +\frac{1}{\gamma} \tilde{\theta}\left(s_{j-1}+\frac{\gamma z_{j}^{6} S_{j}^{T} S_{j}}{2 c_{j}^{2}}-\dot{\hat{\theta}}\right)+\frac{j}{2}+\sum_{i=1}^{j} \frac{\left(c_{i}^{2}+\delta_{i}^{2}\right)}{2} .
\end{aligned}
$$


We choose virtual control variable $\alpha_{j}$ as

$$
\alpha_{j}=-\left(k_{j}+1\right) z_{j}-\frac{z_{j}^{3} S_{j}^{T} S_{j}}{2 c_{j}^{2}} \hat{\theta}-\frac{z_{j}^{3}}{2}+B_{j},
$$

and

$$
\varsigma_{j}=\varsigma_{j-1}+\frac{\gamma z_{j}^{6} S_{j}^{T} S_{j}}{2 c_{j}^{2}} .
$$

Taking (20) and (21) into (19) yields

$$
\mathcal{L} V_{j} \leq-\sum_{i=1}^{j} k_{i} z_{i}^{4}+\frac{1}{\gamma} \tilde{\theta}\left(\varsigma_{j}-\dot{\hat{\theta}}\right)+\frac{1}{4} z_{j+1}^{4}+\frac{j}{2}+\sum_{i=1}^{j} \frac{\left(c_{i}^{2}+\delta_{i}^{2}\right)}{2} .
$$

\section{Step $n$.}

$$
\begin{aligned}
d z_{n}= & d x_{n}-d \alpha_{n-1} \\
= & \left(u+\varphi_{n}\right) d t+g_{n}^{T} d w-d \alpha_{n-1} \\
= & {\left[u(t)+\varphi_{n}-\sum_{i=1}^{n-1} \frac{\partial \alpha_{n-1}}{\partial x_{i}} \varphi_{i}-\frac{1}{2} \sum_{i, m=1}^{n-1} \frac{\partial^{2} \alpha_{n-1}}{\partial x_{i} \partial x_{m}} g_{i}^{T} g_{m}-B_{n}\right] d t } \\
& +\left(g_{n}^{T}-\sum_{i=1}^{n-1} \frac{\partial \alpha_{n-1}}{\partial x_{i}} g_{i}^{T}\right) d w,
\end{aligned}
$$

where $B_{n}=\frac{\partial \alpha_{n-1} \dot{\hat{\theta}}}{\partial \hat{\theta}}+\sum_{i=1}^{n-1} \frac{\partial \alpha_{n-1}}{\partial x_{i}} x_{i+1}+\sum_{i=0}^{n-1} \frac{\partial \alpha_{n-1}}{\partial y_{d}^{(i)}} y_{d}^{(i+1)}$.

Select the following symmetric positive-definite Lyapunov function

$$
V_{n}=V_{n-1}+\frac{1}{4} z_{n}^{4}
$$

There holds

$$
\begin{aligned}
\mathcal{L} V_{n}= & \mathcal{L} V_{n-1}+z_{n}^{3}\left[u(t)+\varphi_{n}-\sum_{i=1}^{n-1} \frac{\partial \alpha_{n-1}}{\partial x_{i}} \varphi_{i}-\frac{1}{2} \sum_{i, m=1}^{n-1} \frac{\partial^{2} \alpha_{n-1}}{\partial x_{i} \partial x_{m}} g_{i}^{T} g_{m}-B_{n}\right] \\
& +\frac{3}{2} z_{n}^{2}\left(g_{n}^{T}-\sum_{i=1}^{n-1} \frac{\partial \alpha_{n-1}}{\partial x_{i}} g_{i}^{T}\right)^{2} .
\end{aligned}
$$

Similar to (16), the inequality can be derived as

$$
\frac{3}{2} z_{n}^{2}\left(g_{n}^{T}-\sum_{i=1}^{n-1} \frac{\partial \alpha_{n-1}}{\partial x_{i}} g_{i}^{T}\right)^{2} \leq \frac{9}{8} z_{n}^{4}\left(g_{n}^{T}-\sum_{i=1}^{n-1} \frac{\partial \alpha_{n-1}}{\partial x_{i}} g_{i}^{T}\right)^{4}+\frac{1}{2} .
$$

Substituting (24) into (23), we obtain

$$
\mathcal{L} V_{n} \leq \mathcal{L} V_{n-1}+z_{n}^{3}\left[u(t)+A_{n}-B_{n}\right]+\frac{1}{2},
$$

where $A_{n}=\varphi_{n}-\sum_{i=1}^{n-1} \frac{\partial \alpha_{n-1}}{\partial x_{i}} \varphi_{i}-\frac{1}{2} \sum_{i, m=1}^{n-1} \frac{\partial^{2} \alpha_{n-1}}{\partial x_{i} \partial x_{m}} g_{i}^{T} g_{m}+\frac{9}{8} z_{n}\left(g_{n}^{T}-\sum_{i=1}^{n-1} \frac{\partial \alpha_{n-1}}{\partial x_{i}} g_{i}^{T}\right)^{4}$.

Now, function $A_{n}$ can be approximated as $A_{n}=W_{n}^{T} S_{n}\left(X_{n}\right)+\xi_{n}\left(X_{n}\right)$, where $\xi_{n}\left(X_{n}\right)$ is the approximation error, $\left\|\xi_{n}\left(X_{n}\right)\right\| \leq \delta_{n}$, and $\delta_{n}>0$. 
Using Young's Inequality, we get

$$
\begin{aligned}
z_{n}^{3} A_{n} & =z_{n}^{3} W_{n}^{T} S_{i}+z_{n}^{3} \xi_{n} \\
& \leq \frac{z_{n}^{6} S_{n}^{T} S_{n}}{2 c_{n}^{2}} \theta+\frac{c_{n}^{2}}{2}+\frac{z_{n}^{6}}{2}+\frac{\delta_{n}^{2}}{2}
\end{aligned}
$$

where $c_{n}>0$.

Substituting (26) into (25), we get

$$
\begin{aligned}
\mathcal{L} V_{n} \leq & \mathcal{L} V_{n-1}+z_{n}^{3}\left[u(t)+\frac{z_{n}^{3} S_{n}^{T} S_{n}}{2 c_{n}^{2}} \theta+\frac{z_{n}^{3}}{2}-B_{n}\right]+\frac{1}{2}+\frac{\left(c_{n}^{2}+\delta_{n}^{2}\right)}{2} \\
\leq & -\sum_{i=1}^{n-1} k_{i} z_{i}^{4}+z_{n}^{3}\left[u(t)+\frac{1}{4} z_{n}+\frac{z_{n}^{3} S_{n}^{T} S_{n}}{2 c_{n}^{2}} \hat{\theta}+\frac{z_{n}^{3}}{2}-B_{n}\right] \\
& +\frac{1}{\gamma} \tilde{\theta}\left(s_{n-1}+\frac{\gamma z_{n}^{6} S_{n}^{T} S_{n}}{2 c_{n}^{2}}-\dot{\hat{\theta}}\right)+\frac{n}{2}+\sum_{i=1}^{n} \frac{\left(c_{i}^{2}+\delta_{i}^{2}\right)}{2} .
\end{aligned}
$$

We choose virtual control variable $\alpha_{n}$ as

$$
\alpha_{n}=-\left(k_{n}+\frac{1}{4}\right) z_{n}-\frac{z_{n}^{3} S_{n}^{T} S_{n}}{2 c_{n}^{2}} \hat{\theta}-\frac{z_{n}^{3}}{2}+B_{n}
$$

and

$$
\varsigma_{n}=\varsigma_{n-1}+\frac{\gamma z_{n}^{6} S_{n}^{T} S_{n}}{2 c_{n}^{2}} .
$$

Substituting (28) and (29) into (27), we can obtain

$$
\mathcal{L} V_{n} \leq-\sum_{i=1}^{n} k_{i} z_{i}^{4}+z_{n}^{3}\left[u(t)-\alpha_{n}\right]+\frac{1}{\gamma} \tilde{\theta}\left(\varsigma_{n}-\dot{\hat{\theta}}\right)+\frac{n}{2}+\sum_{i=1}^{n} \frac{\left(c_{i}^{2}+\delta_{i}^{2}\right)}{2} .
$$

With the view of reducing the load of computation and transmission, we introduce a dynamic variable in designing the event-triggered condition during the controller design process. The condition can be designed in such a way that

$$
t_{k+1}=\inf \left\{t>t_{k} \mid\left\|u(t)-\alpha_{n}\right\| \geq \eta_{r}+h\right\},
$$

where $\eta_{r}$ is an internal dynamic variable to be designed later; $h>0$ is a design parameter; $t_{0}=0$; and $t_{k}$ is the controller update time.

From (31), for $\forall t \in\left[t_{k}, t_{k+1}\right)$, there holds

$$
\left\|u(t)-\alpha_{n}\right\| \leq \eta_{r}+h,
$$

and further gets

$$
u(t)=\alpha_{n}-\sigma(t)\left(\eta_{r}+h\right),
$$

where $\sigma\left(t_{k}\right)=0,|\sigma(t)| \leq 1, \sigma\left(t_{k+1}\right)= \pm 1$.

According to the nature of hyperbolic tangent function $\tanh (B)$, we can see $0 \leq|B|-B \tanh \left(\frac{B}{\varepsilon}\right) \leq 0.2785 \varepsilon$, where $\varepsilon>0$ and $B \in \mathbb{R}$. Then, $\omega(t)$ is used to eliminate term $\eta_{r}+h$ in (33). Design $\omega(t)$ as

$$
\omega(t)=\alpha_{n}-\left(\eta_{r}+h\right) \tanh \left(\frac{z_{n}^{3}\left(\eta_{r}+h\right)}{\varepsilon}\right) .
$$


Now, the event-triggered condition and controller have been redesigned for system (1) as follows:

$$
\begin{gathered}
t_{k+1}=\inf \left\{t>t_{k} \mid\|u(t)-\omega(t)\| \geq \eta_{r}+h\right\}, \\
u(t)=\omega\left(t_{k}\right), t \in\left[t_{k}, t_{k+1}\right) .
\end{gathered}
$$

Remark 2. In [28], the authors study the dynamic event-triggered tracking control for deterministic nonlinear systems. Compared with [28], stochastic disturbances are considered in this paper. Obviously, such stochastic nonlinear systems are more practical and more general [40-42].

Choose a Lyapunov function $\bar{V}=V_{n}+\eta_{r}$ with $\eta_{r} \geq 0$. Then, from Lemma 1, it follows that

$$
\begin{aligned}
\mathcal{L} \bar{V}= & \mathcal{L} V_{n}+\dot{\eta}_{r} \\
\leq & -\sum_{i=1}^{n} k_{i} z_{i}^{4}+z_{n}^{3}\left[u(t)-\alpha_{n}\right]+\frac{1}{\gamma} \tilde{\theta}\left(\varsigma_{n}-\dot{\hat{\theta}}\right)+\frac{n}{2}+\sum_{i=1}^{n} \frac{\left(c_{i}^{2}+\delta_{i}^{2}\right)}{2}+\dot{\eta}_{r} \\
= & -\sum_{i=1}^{n} k_{i} z_{i}^{4}-\eta_{r}+z_{n}^{3}[u(t)-\mathcal{\omega}(t)]-z_{n}^{3}\left(\eta_{r}+h\right) \tanh \left(\frac{z_{n}^{3}\left(\eta_{r}+h\right)}{\varepsilon}\right) \\
& +\frac{1}{\gamma} \tilde{\theta}\left(\varsigma_{n}-\dot{\hat{\theta}}\right)+\frac{n}{2}+\sum_{i=1}^{n} \frac{\left(c_{i}^{2}+\delta_{i}^{2}\right)}{2}+\eta_{r}+\dot{\eta}_{r} .
\end{aligned}
$$

From event-triggered condition (35), we have $u(t)=\omega(t)-\sigma(t)\left(\eta_{r}+h\right)$, and the following inequality is obtained

$$
\begin{aligned}
\mathcal{L} \bar{V} \leq & -\sum_{i=1}^{n} k_{i} z_{i}^{4}-\eta_{r}-z_{n}^{3} \sigma(t)\left(\eta_{r}+h\right)-z_{n}^{3}\left(\eta_{r}+h\right) \tanh \left(\frac{z_{n}^{3}\left(\eta_{r}+h\right)}{\varepsilon}\right) \\
& +\frac{1}{\gamma} \tilde{\theta}\left(\varsigma_{n}-\dot{\hat{\theta}}\right)+\frac{n}{2}+\sum_{i=1}^{n} \frac{\left(c_{i}^{2}+\delta_{i}^{2}\right)}{2}+\eta_{r}+\dot{\eta}_{r} \\
= & -\sum_{i=1}^{n} k_{i} z_{i}^{4}-\eta_{r}-z_{n}^{3} \sigma(t)\left(\eta_{r}+h\right)-\left|z_{n}^{3}\left(\eta_{r}+h\right)\right|+\left|z_{n}^{3}\left(\eta_{r}+h\right)\right| \\
& -z_{n}^{3}\left(\eta_{r}+h\right) \tanh \left(\frac{z_{n}^{3}\left(\eta_{r}+h\right)}{\varepsilon}\right)+\frac{1}{\gamma} \tilde{\theta}\left(\varsigma_{n}-\dot{\hat{\theta}}\right)+\sum_{i=1}^{n} \frac{\left(c_{i}^{2}+\delta_{i}^{2}\right)}{2} \\
& +\frac{n}{2}+\eta_{r}+\dot{\eta}_{r} \\
\leq & -\sum_{i=1}^{n} k_{i} z_{i}^{4}-\eta_{r}-z_{n}^{3} \sigma(t)\left(\eta_{r}+h\right)-\left|z_{n}^{3}\left(\eta_{r}+h\right)\right|+0.2785 \varepsilon \\
& +\frac{1}{\gamma} \tilde{\theta}\left(\varsigma_{n}-\dot{\hat{\theta}}\right)+\sum_{i=1}^{n} \frac{\left(c_{i}^{2}+\delta_{i}^{2}\right)}{2}+\frac{n}{2}+\eta_{r}+\dot{\eta}_{r} \\
\leq & -\sum_{i=1}^{n} k_{i} z_{i}^{4}-\eta_{r}+\frac{1}{\gamma} \tilde{\theta}\left(\varsigma_{n}-\dot{\hat{\theta}}\right)+0.2785 \varepsilon+\sum_{i=1}^{n} \frac{\left(c_{i}^{2}+\delta_{i}^{2}\right)}{2}+\frac{n}{2} \\
& +\eta_{r}+\dot{\eta}_{r} .
\end{aligned}
$$

In order to assure the stochastic stability of the closed-loop system, we design $\eta_{r}+\dot{\eta}_{r} \leq$ $-k \eta_{r} \leq 0$, where $k>0$, and we get

$$
\eta_{r} \leq-k \eta_{r}-\eta_{r}
$$


From (35), for $\forall t \in\left[t_{k}, t_{k+1}\right)$, it is obvious that $\|u(t)-\mathfrak{\omega}(t)\| \leq \eta_{r}+h$, i.e.,

$$
-\eta_{r} \leq h-\|u(t)-\omega(t)\| .
$$

Substituting (40) into (39), we get

$$
\dot{\eta}_{r} \leq-k \eta_{r}+h-\|u(t)-\omega(t)\| .
$$

Finally, based on (41), we choose

$$
\dot{\eta}_{r}=-k \eta_{r}+h-\|u(t)-\omega(t)\|,
$$

and design the adaptive law as

$$
\dot{\hat{\theta}}=\varsigma_{n}-\gamma \hat{\theta}
$$

Remark 3. Similar to [15], according to the event-triggered condition (35) with (42) and $\eta_{r} \geq 0$, we can obtain that $\eta_{r}+\left(h-\left\|u(t)-\mathfrak{\omega}\left(t_{k+1}\right)\right\|\right) \geq 0$, which implies that $\eta_{r}$ is bounded by $0 \leq \eta_{r} \leq \frac{h}{k}$. We can also observe that the lower bound on the execution time of DETM is larger than that of SETM, which means the communication resources can be further saved.

Now, substituting (42) and (43) into (38), we get

$$
\mathcal{L} \bar{V} \leq-\sum_{i=1}^{n} k_{i} z_{i}^{4}-\eta_{r}+0.2785 \varepsilon+\tilde{\theta} \hat{\theta}+\frac{n}{2}+\sum_{i=1}^{n} \frac{\left(c_{i}^{2}+\delta_{i}^{2}\right)}{2} .
$$

By Lemma 2, we have $\tilde{\theta} \hat{\theta} \leq-\frac{1}{2} \tilde{\theta}^{2}+\frac{1}{2} \theta^{2}$ and

$$
\begin{aligned}
\mathcal{L} \bar{V} & \leq-\sum_{i=1}^{n} k_{i} z_{i}^{4}-\eta_{r}-\frac{1}{2} \tilde{\theta}^{2}+0.2785 \varepsilon+\frac{1}{2} \theta^{2}+\frac{n}{2}+\sum_{i=1}^{n} \frac{\left(c_{i}^{2}+\delta_{i}^{2}\right)}{2} \\
& =-N \bar{V}+\Lambda
\end{aligned}
$$

where $N=\min \left\{4 k_{1}, \ldots, 4 k_{n}, \gamma, 1\right\}, \Lambda=0.2785 \varepsilon+\frac{1}{2} \theta^{2}+\frac{n}{2}+\sum_{i=1}^{n} \frac{\left(c_{i}^{2}+\delta_{i}^{2}\right)}{2}$. From (45), it can be obtained that

$$
\frac{d E[\bar{V}(t)]}{d t} \leq-N E[\bar{V}(t)]+\Lambda .
$$

Furthermore, inequality (46) satisfies

$$
\begin{aligned}
E[\bar{V}(t)] & \leq\left[\bar{V}(0)-\frac{\Lambda}{N}\right] e^{-N t}+\frac{\Lambda}{N} \\
& \leq \bar{V}(0) e^{-N t}+\frac{\Lambda}{N^{\prime}}, \quad \forall t>0,
\end{aligned}
$$

and

$$
0 \leq E[\bar{V}(t)] \leq \bar{V}(0)+\frac{\Lambda}{N^{\prime}}, \forall t>0,
$$

where $\bar{V}(0)=\sum_{j=1}^{n} \frac{1}{4} z_{j}^{4}(0)+\frac{1}{2 \gamma} \tilde{\theta}^{2}(0)+\eta_{r}(0)$. From (48), the following compact set $\Omega$ can be obtained

$$
\Omega=\left\{z_{j}, \tilde{\theta} \mid \sum_{j=1}^{n} E\left[\left\|z_{j}\right\|^{4}\right] \leq 4 \bar{V}(0)+4 \frac{\Lambda}{N},\|\tilde{\theta}\| \leq \sqrt{2 \gamma\left[\bar{V}(0)+\frac{\Lambda}{N}\right]}\right\} .
$$


By combining (49) with Definition 2, it can be obtained that variable $z_{j}$ and parameter $\tilde{\theta}$ eventually converge to the compact set $\Omega$. Therefore, it has been proven that the signals of the closed-loop system are SGUUB in the fourth moment, and $\tilde{\theta}$ is SGUUB in mean square.

Based on the above discussion, it is straightforward to obtain the following results.

Theorem 1. Consider stochastic nonlinear system (1) with dynamic event-triggered condition (35), virtual controller (34), actual controller (36), and adaptive law (43). All the signals of the closed-loop system are fourth moment SGUUB.

According to Theorem 1, we can also deduce that the designed DETC can eliminate the Zeno phenomenon. From (34), we can derive the boundedness and continuity of $\dot{\omega}(t)$, which means there has a constant $m$ such that $|\dot{\omega}(t)| \leq m$. In view of (34), let $\beta(t)=u(t)-\omega(t)$ for $\forall t \in\left[t_{k}, t_{k+1}\right)$, and then

$$
\left|\frac{d}{d t} \beta(t)\right|=\operatorname{sign}(\dot{\beta}) \dot{\beta} \leq|\dot{\omega}(t)| \leq m .
$$

Moreover, it is noted that $\beta\left(t_{k}\right)=0$ and $\lim _{t \rightarrow t_{k+1}} \beta(t)=\eta_{r}+h>0$. Thus, there exists

$$
t^{*}=t_{k+1}-t_{k} \geq \frac{\eta_{r}+h}{m}>0 .
$$

This implies that the Zeno phenomenon is excluded.

\section{Numerical Simulation}

Now, we take two simulation examples to show the validity of the proposed control method.

Example 1. Consider the following stochastic nonlinear system:

$$
\left\{\begin{array}{l}
d x_{1}=\left[x_{2}+\varphi_{1}\left(x_{1}\right)\right] d t+g_{1}^{T}\left(x_{1}\right) d w \\
d x_{2}=\left[u(t)+\varphi_{2}\left(x_{1}, x_{2}\right)\right] d t+g_{2}^{T}\left(x_{1}, x_{2}\right) d w \\
y=x_{1}
\end{array}\right.
$$

where $\varphi_{1}\left(x_{1}\right)=x_{1}^{2}, g_{1}\left(x_{1}\right)=0.1 \cos \left(x_{1}\right), \varphi_{2}\left(x_{1}, x_{2}\right)=x_{1} x_{2}^{2}, g_{2}\left(x_{1}, x_{2}\right)=\sin \left(x_{1} x_{2}\right)$. The control objective is to make $y$ track $y_{d}=0.5 \cos (2 \pi t)$. Under the control design method proposed above, the controller and the adaptive law are designed as

$$
\left\{\begin{array}{l}
\alpha_{1}=-\left(k_{1}+\frac{3}{4}\right) z_{1}-\frac{z_{1}^{3} S_{1}^{T} S_{1}}{2 c_{1}^{2}} \hat{\theta}-\frac{z_{1}^{3}}{2}+\dot{y}_{d} \\
\alpha_{2}=-\left(k_{2}+\frac{1}{4}\right) z_{2}-\frac{z_{2}^{3} S_{2}^{T} S_{2}}{2 c_{2}^{2}} \hat{\theta}-\frac{z_{2}^{3}}{2}+B_{2} \\
B_{2}=\frac{\partial \alpha_{1}}{\partial \hat{\theta}} \dot{\hat{\theta}}+\frac{\partial \alpha_{1}}{\partial x_{1}} x_{2}+\frac{\partial \alpha_{1}}{\partial y_{d}} \dot{y}_{d}+\frac{\partial \alpha_{1}}{\partial \dot{y}_{d}} \ddot{y}_{d} \\
u(t)=\omega\left(t_{k}\right), k \in Z^{+} \\
\dot{\hat{\theta}}=\varsigma_{2}-\gamma \hat{\theta}
\end{array}\right.
$$

In this paper, the DETM we studied is

$$
\left\{\begin{array}{l}
t_{k+1}=\inf \left\{t>t_{k} \mid\|u(t)-\omega(t)\| \geq \eta_{r}+h\right\}, \\
\dot{\eta}_{r}=-k \eta_{r}+(h-\|u(t)-\mathcal{\omega}(t)\|), \\
\mathcal{\omega}(t)=\alpha_{2}-\left(\eta_{r}+h\right) \tanh \left(\frac{z_{2}^{3}\left(\eta_{r}+h\right)}{\epsilon}\right) .
\end{array}\right.
$$


To make a comparison, the simulations are also constructed with SETM

$$
\left\{\begin{array}{l}
t_{k+1}=\inf \left\{t>t_{k} \mid\|u(t)-\omega(t)\| \geq h\right\}, \\
\omega(t)=\alpha_{2}-h \tanh \left(\frac{z_{2}^{3} h}{\epsilon}\right) .
\end{array}\right.
$$

In this simulation, we choose the initial conditions as $\hat{\theta}(0)=0, \eta_{r}(0)=0, x_{1}(0)=1$, $x_{2}(0)=-5$. The parameters are selected as $k_{1}=45, k_{2}=40, c_{1}=1, c_{2}=2, \gamma=10$, $h=4, \varepsilon=1$, and $k=0.5$. The radial basis functions are chosen as $S_{j}=\exp \left[-\frac{\left\|x_{j}-\mu_{j}\right\|^{2}}{2 \sigma_{j}^{2}}\right]$ with $\mu_{j}=[-1,-0.5,0,0.5,1]$ and widths $\sigma_{j}=2$.

Figure 1 shows the trajectories of output signal $y$ and reference signal $y_{d}$. We can see that the output signal soon tracks the reference signal under the two control mechanisms. Further, the simulation of tracking errors under DETM and SETM is given in Figure 2, which further illustrates the tracking performance of the system under different mechanisms. The parameter adaptive laws $\hat{\theta}$ is bounded which is depicted in Figure 3. From Figure 4, it can be seen that the threshold under DETM is adaptively adjusted through a bounded dynamic variable, while the threshold under SETM is a fixed value. Figure 5 gives the triggered time interval under both mechanisms, and it can be seen that the Zeno phenomenon is avoided. Table 1 gives the triggering times under both mechanisms when $h$ takes different values, which further illustrates that DETM saves more resources than SETM.

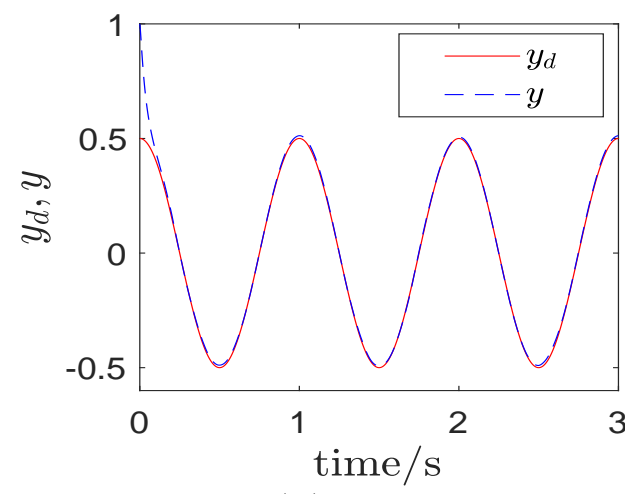

(a) DETM

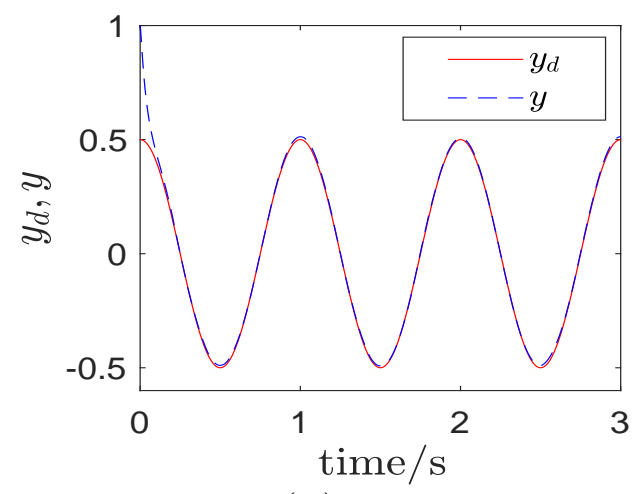

(b) SETM

Figure 1. The trajectories of output signal $y$ and reference signal $y_{d}$.

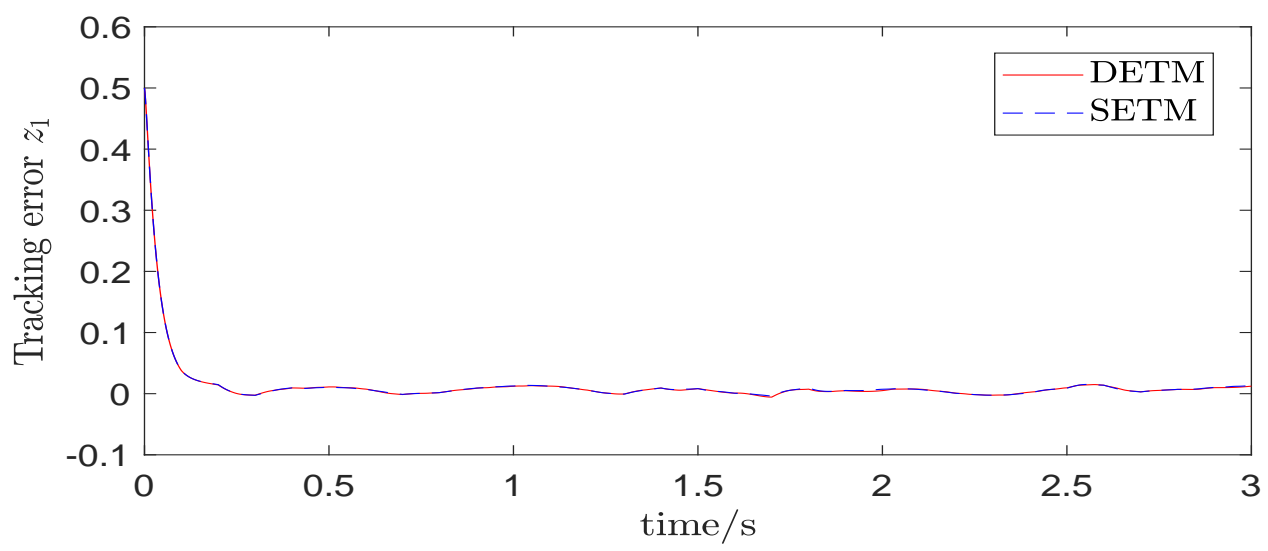

Figure 2. Tracking errors under DETM and SETM. 


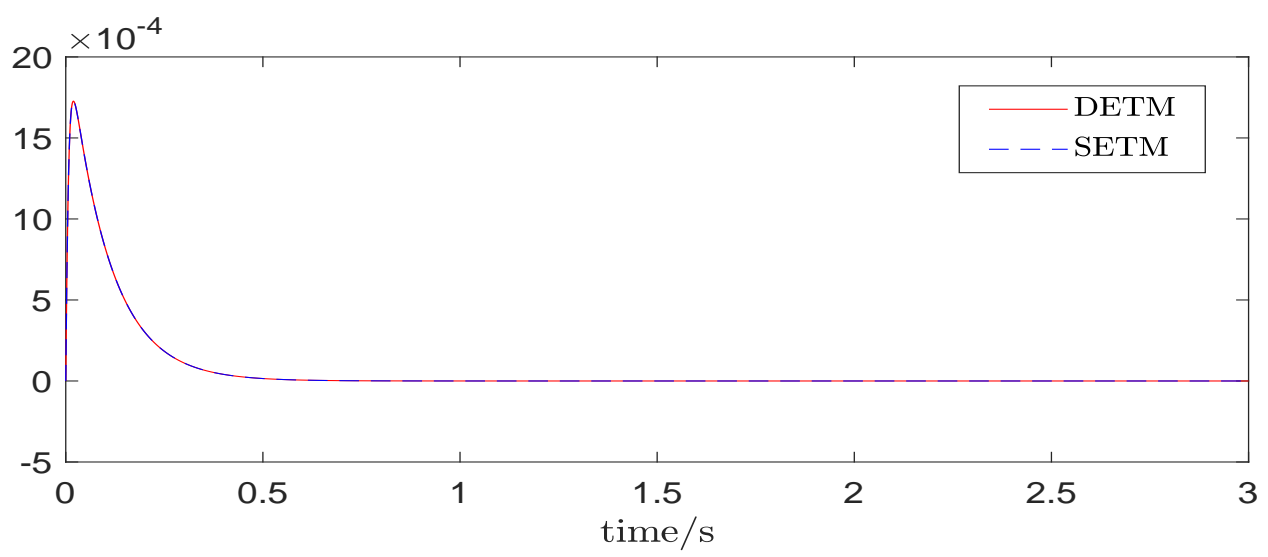

Figure 3. The trajectories of $\hat{\theta}$ under DETM and SETM.

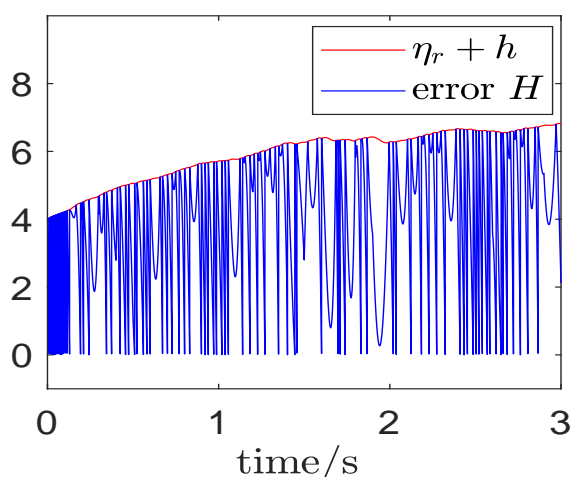

(a) $H=\eta_{r}+h-\|u(t)-\varpi(t)\|$

Figure 4. Event-triggered errors.

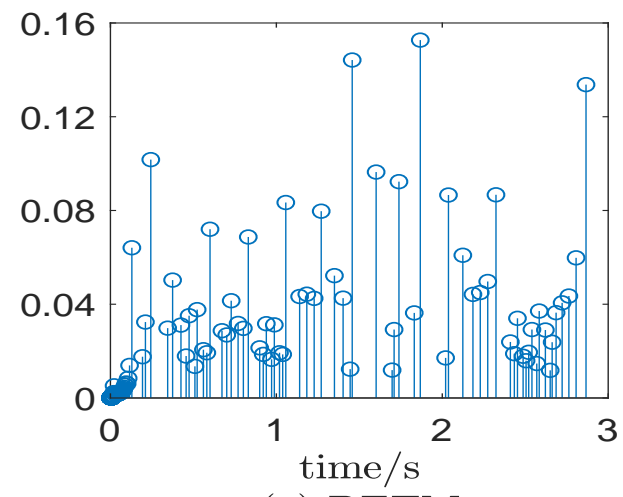

(a) DETM

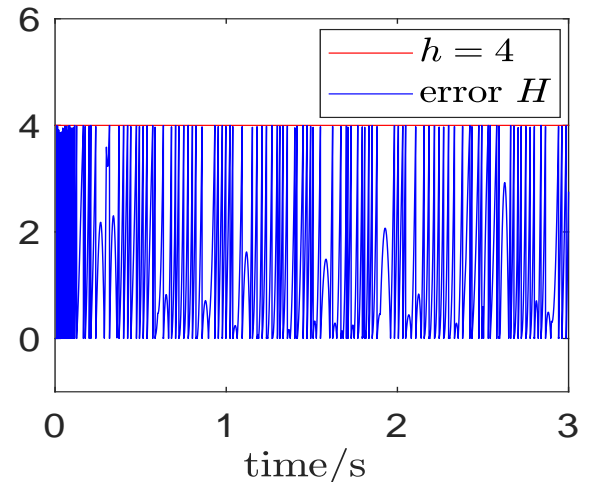

(b) $H=h-\|u(t)-\varpi(t)\|$

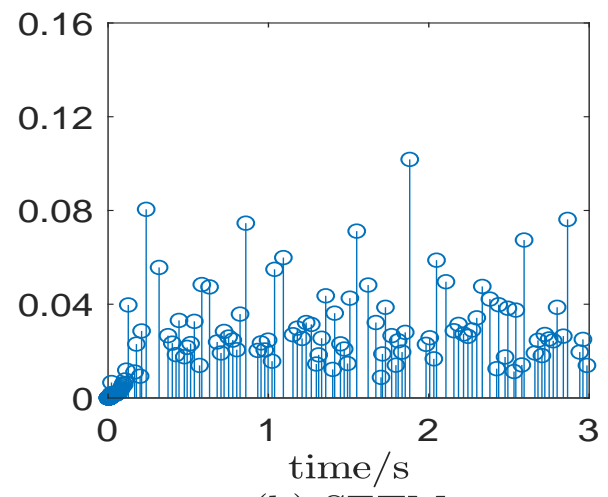

(b) SETM

Figure 5. Triggered time interval $t_{k}-t_{k-1}$.

Table 1. Inter-event times.

\begin{tabular}{ccccc}
\hline & $\mathbf{h}=\mathbf{4}$ & $\mathbf{h}=\mathbf{3}$ & $\mathbf{h}=\mathbf{2}$ & $\mathbf{h}=\mathbf{1}$ \\
\hline DETM & 226 & 267 & 325 & 487 \\
\hline SETM & 254 & 297 & 362 & 559 \\
\hline
\end{tabular}

Remark 4. From Table 1, we can see that when h takes smaller values, the triggering times of DETM are obviously less than that of SETM. This is mainly because $\eta_{r}$ plays a key role in the threshold of DETM only when $h$ takes a small value. When $h$ takes a large value, DETM is similar to SETM. 
Example 2. To further demonstrate the effectiveness of the control method, the single-link robot arm system [7] operating in noise environments is considered. When the system is without noise, the model is

$$
\ddot{\theta}(t)=\frac{1}{J} u(t)-\frac{M g L}{J} \sin (\theta(t))-\frac{R}{J} \dot{\theta}(t),
$$

where $\theta(t)$ and $\dot{\theta}(t)$ denote the angle and angular velocity of the robot arm, respectively; J represents the moment of inertia; $M$ denotes the mass of the load; $L$ represents the value of the length; $g$ is the acceleration of gravity; $R$ is damping coefficient. The values of these relative parameters are $J=1$ $\mathrm{kg} \cdot \mathrm{m}^{2}, M=1 \mathrm{~kg}, g=10 \mathrm{~N} / \mathrm{kg}, L=0.5 \mathrm{~m}$, and $R=2 \mathrm{Nm} \cdot \mathrm{s} / \mathrm{rad}$.

Take noise disturbance into consideration and define $x_{1}=\theta(t), x_{2}=\dot{\theta}(t)$. Equation (55) can be reformulated as

$$
\left\{\begin{array}{l}
d x_{1}=x_{2} d t+g_{1}^{T}\left(x_{1}\right) d w \\
d x_{2}=\left[\frac{1}{J} u(t)-\frac{M g L}{J} \sin \left(x_{1}\right)-\frac{R}{J} x_{2}\right] d t+g_{2}^{T}\left(x_{1}, x_{2}\right) d w \\
y=x_{1} .
\end{array}\right.
$$

where $\varphi_{1}\left(x_{1}\right)=0, g_{1}\left(x_{1}\right)=x_{1}^{2}, \varphi_{2}\left(x_{1}, x_{2}\right)=-2 x_{2}-5 \sin \left(x_{1}\right), g_{2}\left(x_{1}, x_{2}\right)=x_{2} \cos \left(x_{1}\right)$, and the tracking signal is $y_{d}=0.5 \sin (2 \pi t)+0.5 \sin (\pi t)$.

According to the aforementioned analysis, the designed DETC with NNs for this example are the same as Example 1. Similarly, we choose the initial conditions as $\hat{\theta}(0)=0$, $\eta_{r}(0)=0, x_{1}(0)=0.8, x_{2}(0)=-5$ and select the parameters as $k_{1}=45, k_{2}=30, c_{1}=1$, $c_{2}=1, \gamma=10, h=5, \varepsilon=1, k=0.5$.

As can be seen in Figure 6, the output signal $y$ can track $y_{d}$. In Figure 7, tracking error $z_{1}$ converges to zero within a very small range. The trajectory of adaptive parameter and dynamically threshold are shown in Figures 8 and 9, respectively. It also can be seen from Figure 9 that the threshold is dynamically adjustable under DETM. Moreover, the dynamic variable $\eta_{r}$ is bounded. From the triggered time interval in Figure 10, we deduce that the Zeno phenomenon is avoided under DETM. The simulation verifies the obtained theoretical results.

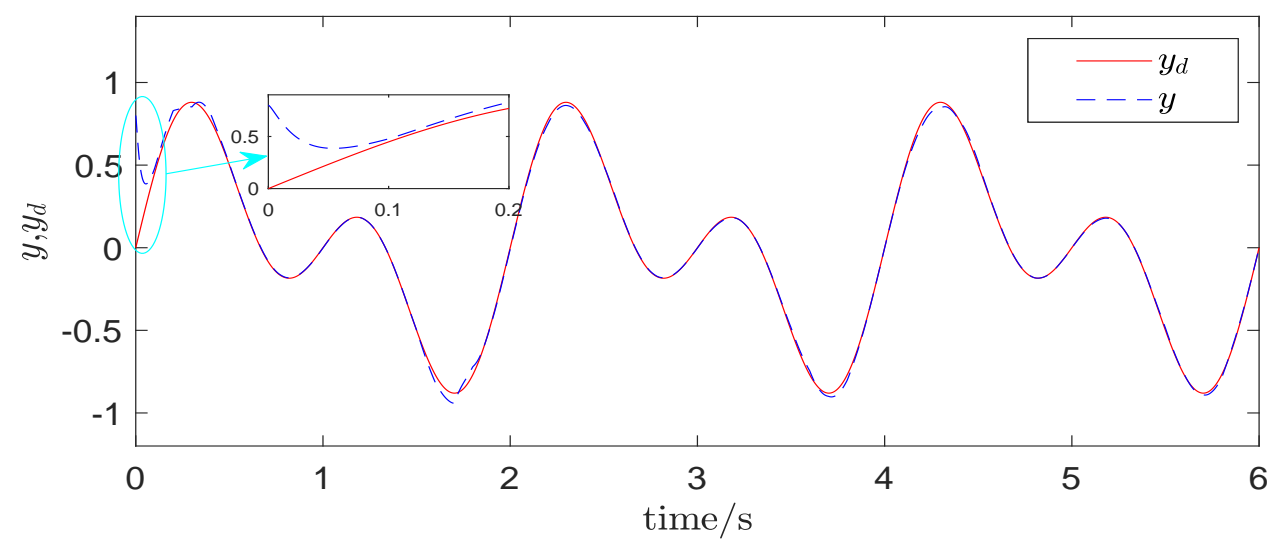

Figure 6. The trajectories of output signal and reference signal. 


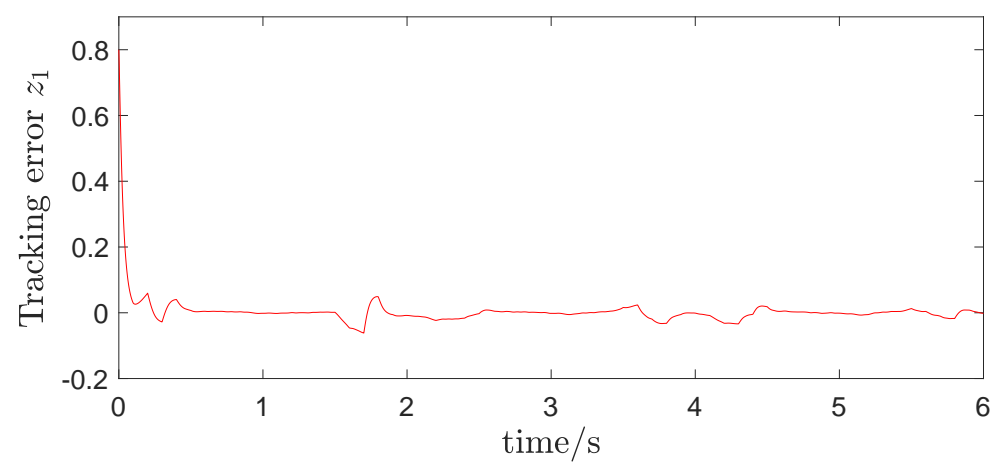

Figure 7. Tracking error.

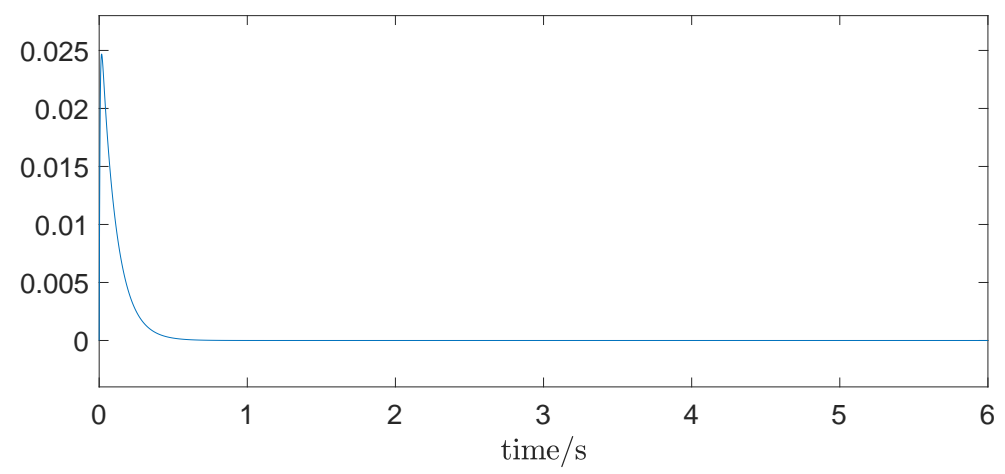

Figure 8. The trajectory of $\hat{\theta}$.

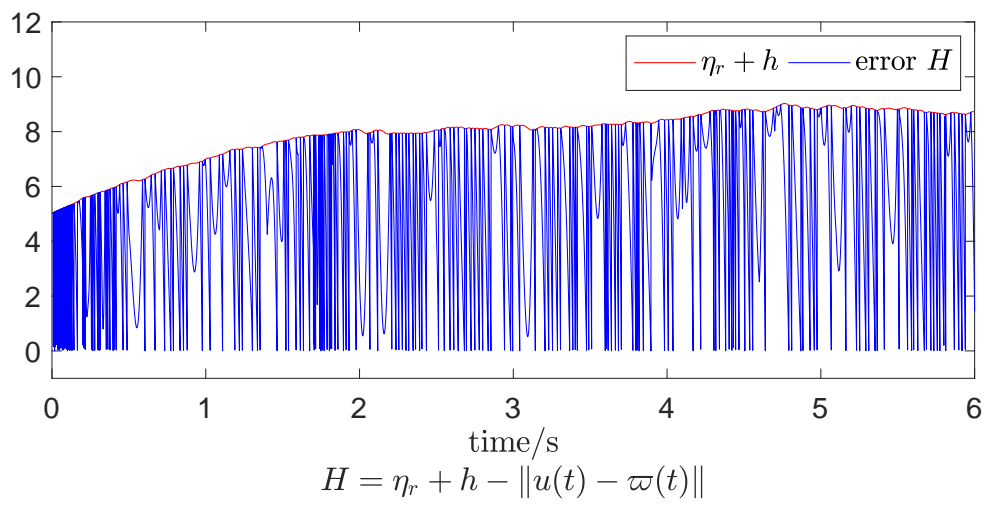

Figure 9. Event-triggered error.

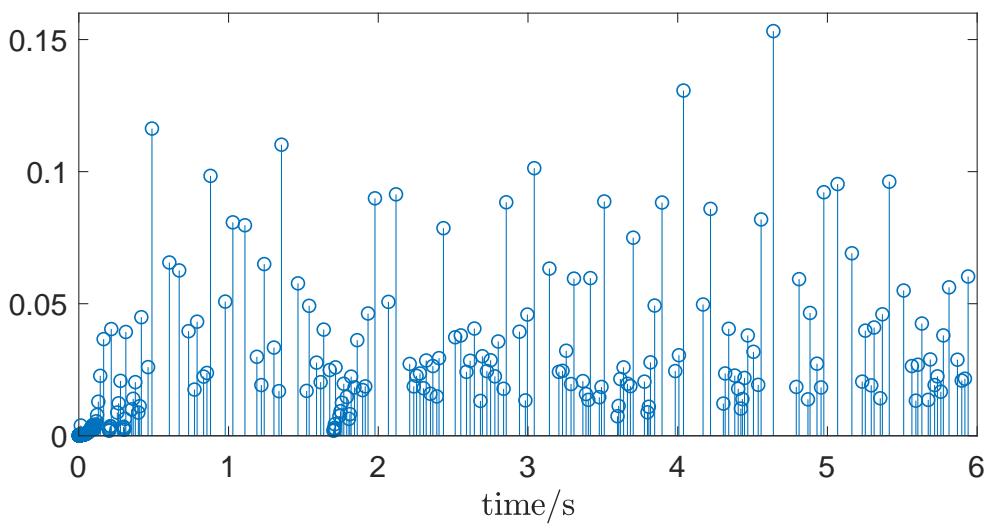

Figure 10. Triggered time interval $t_{k}-t_{k-1}$. 


\section{Conclusions}

For a class of unknown stochastic nonlinear strict-feedback systems, a dynamic eventtriggered design method is investigated in this paper. Combining adaptive backstepping and NNs, an adaptive DETC is designed by adding a dynamic variable to the triggering threshold. The theoretical results ensure that the signals of the closed-loop system are SGUUB in the sense of the fourth moment, and the final simulation shows that DETM can save resources more efficiently than SETM.

Author Contributions: Supervision, J.L.; Writing—original draft, Y.F.; Writing—review \& editing, S.W. and X.L. All authors have read and agreed to the published version of the manuscript.

Funding: This work was supported in part by National Natural Science Foundation of China (61673014), Natural Fundation of Xianyang Normal University under Grants (12XSYK011) and Special Scientifific Research Plan Project of Shaanxi Provincial Department of Education (No. 21JK0488).

Institutional Review Board Statement: Not applicable.

Informed Consent Statement: Not applicable.

Conflicts of Interest: The authors declare no conflict of interest.

\section{References}

1. Åarzén, K.E. A simple event-based PID controller. IFAC Proc. Vol. 1999, 32, 8687-8692. [CrossRef]

2. Åström, K.J.; Bernhardsson, B. Comparison of periodic and event based sampling for first-order stochastic systems. IFAC Proc. Vol. 1999, 32, 5006-5011. [CrossRef]

3. Tabuada, P. Event-triggered real-time scheduling of stabilizing control tasks. IEEE Trans. Autom. Control. 2007, 52, 1680-1685. [CrossRef]

4. Tallapragada, P.; Chopra, N. On event triggered tracking for nonlinear systems. IEEE Trans. Autom. Control. 2013, 58, 2343-2348. [CrossRef]

5. Xing, L.T.; Wen, C.Y.; Liu, Z.T.; Su, H.Y.; Cai, J.P. Event-triggered adaptive control for a class of uncertain nonlinear systems. IEEE Trans. Autom. Control. 2017, 62, 2071-2076. [CrossRef]

6. Xing, L.T.; Wen, C.Y.; Liu, Z.T.; Su, H.Y.; Cai, J.P. Event-triggered output feedback control for a class of uncertain nonlinear systems. IEEE Trans. Autom. Control. 2019, 64, 290-297. [CrossRef]

7. He, M.; Li, J.M. Event-triggered adaptive tracking control for a class of uncertain stochastic nonlinear systems with Markov jumping parameters. Int. J. Adapt. Control Signal Process. 2018, 32, 1655-1674. [CrossRef]

8. Liu, C.G.; Liu, X.P.; Wang, H.Q.; Zhou, Y.C.; Lu, S.Y.; Xu, B. Event-triggered adaptive tracking control for uncertain nonlinear systems based on a new funnel function. ISA Trans. 2020, 99, 130-138. [CrossRef] [PubMed]

9. Luo, S.X.; Deng, F.Q. On event-triggered control of nonlinear stochastic systems. IEEE Trans. Autom. Control 2019, 65, 369-375. [CrossRef]

10. Qiu, J.B.; Ma, M.; Wang, T. Event-triggered adaptive fuzzy fault-tolerant control for stochastic nonlinear systems via command filtering. IEEE Trans. Syst. Man Cybern. Syst. 2020. [CrossRef]

11. Li, B.M.; Xia, J.W.; Zhang, H.S.; Shen, H.; Wang, Z. Event-triggered adaptive fuzzy tracking control for stochastic nonlinear systems. J. Frankl. Inst. 2020, 357, 9505-9522. [CrossRef]

12. Lian, Y.X.; Xia, J.W.; Sun, W.; Wang, Z.; Zhang, H.S. Adaptive fuzzy control for non-strict-feedback stochastic uncertain non-linear systems based on event-triggered strategy. IET Control Theory Appl. 2021, 15, 1018-1027. [CrossRef]

13. Hu, Z.H.; Mu, X.W. Event-Triggered Impulsive Control for Nonlinear Stochastic Systems. IEEE Trans. Cybern. 2021. [CrossRef]

14. Zhao, N.N.; Ouyang, X.Y.; Wu, L.B.; Shi, F.R. Event-triggered adaptive prescribed performance control of uncertain nonlinear systems with unknown control directions. ISA Trans. 2020, 99, 130-138. [CrossRef]

15. Girard, A. Dynamic triggering mechanisms for event-triggered control. IEEE Trans. Autom. Control. 2015, 60, 1992-1997. [CrossRef]

16. Tripathy, N.S.; Kar, I.N.; Paul, K. Robust dynamic event-triggered control for linear uncertain system. IFAC-PapersOnLine 2016, 49, 207-212. [CrossRef]

17. Hu, W.F.; Yang, C.H. Consensus of linear multi-agent systems by distributed dynamic event-triggered control. In Proceedings of the 2017 International Workshop on Complex Systems and Networks (IWCSN), Doha, Qatar, 8-10 December 2017; pp. 284-289. [CrossRef]

18. Li, P.F.; Kang, Y.; Zhao, Y.B.; Zhou, J. Dynamic event-triggered control for networked switched linear systems. In Proceedings of the 2017 36th Chinese Control Conference (CCC), Dalian, China, 26-28 July 2017; pp. 7984-7989. [CrossRef]

19. Ge, X.H.; Han, Q.L. Distributed formation control of networked multi-agent systems using a dynamic event-triggered communication mechanism. IEEE Trans. Ind. Electron. 2017, 64, 8118-8127. [CrossRef]

20. Zuo, Z.Q.; Guan, S.Y.; Wang, Y.J.; Li, H.C. Dynamic event-triggered and self-triggered control for saturated systems with anti-windup compensation. J. Frankl. Inst. 2017, 354, 7624-7642. [CrossRef] 
21. Wang, Y.J.; Jia, Z.X.; Zuo, Z.Q. Dynamic event-triggered and self-triggered output feedback control of networked switched linear systems. Neurocomputing 2018, 314, 39-47. [CrossRef]

22. Hu, W.F.; Yang, C.H.; Huang, T.W.; Gui, W.H. A distributed dynamic event-triggered control approach to consensus of linear multiagent systems with directed networks. IEEE Trans. Cybern. 2018, 50, 869-874. [CrossRef] [PubMed]

23. Dolk, V.S.; Borgers, D.P.; Heemels, W. Output-based and decentralized dynamic event-triggered control with guaranteed L-p-gain performance and Zeno-freeness. IEEE Trans. Autom. Control. 2016, 62, 34-49. [CrossRef]

24. Li, H.; Liu, Y.G.; Huang, Y.X. Adaptive stabilization via dynamic event-triggered output feedback for uncertain nonlinear systems. J. Control Theory Appl. 2019, 36, 1871-1878. [CrossRef]

25. Li, F.Z.; Liu, Y.G. Adaptive event-triggered output-feedback controller for uncertain nonlinear systems. Automatica 2020, 117, 109006. [CrossRef]

26. Mu, C.X.; Wang, K.; Qiu, T. Dynamic event-triggering neural learning control for partially unknown nonlinear systems. IEEE Trans. Cybern. 2020. [CrossRef]

27. Zuo, Z.Q.; Xie, P.F.; Wang, Y.J. Output-based dynamic event-triggering control for sensor saturated systems with external disturbance. Appl. Math. Comput. 2020, 374, 125043. [CrossRef]

28. Gao, H.; Song, Y.D.; Wen, C.Y. Event-triggered adaptive neural network controller for uncertain nonlinear system. Inf. Sci. 2020, 506, 148-160. [CrossRef]

29. Wu, J.; Chen, X.M.; Zhao, Q.J.; Li, J.; Wu, Z.G. Adaptive neural dynamic surface control with prespecified tracking accuracy of uncertain stochastic nonstrict-feedback systems. IEEE Trans. Cybern. 2020. [CrossRef] [PubMed]

30. $\mathrm{Wu}$, J.; Sun, Y.B.; Zhao, Q.J. Adaptive asymptotic control of stochastic systems with state delay and unknown control directions. IEEE T Circuits-II 2021. [CrossRef]

31. Seshagiri, S.; Khalil, H.K. Output feedback control of nonlinear systems using RBF neural networks. IEEE Trans. Neural Netw. 2000, 11, 69-79. [CrossRef] [PubMed]

32. Wang, D.; Huang, J. Adaptive neural network control for a class of uncertain nonlinear systems in pure-feedback form. Automatica 2002, 38, 1365-1372. [CrossRef]

33. Li, J.; Chen, W.S.; Li, J.M.; Fang, Y.Q. Adaptive NN output-feedback stabilization for a class of stochastic nonlinear strict-feedback systems. ISA Trans. 2009, 48, 468-475. [CrossRef] [PubMed]

34. Chen, W.S.; Jiao, L.C.; Li, J.; Li, R.H. Adaptive NN backstepping output-feedback control for stochastic nonlinear strict-feedback systems with time-varying delays. IEEE Trans. Syst. Man Cybern B Cybern. 2009, 40, 939-950. [CrossRef]

35. Chen, W.S. Output-feedback adaptive stochastic nonlinear stabilization using neural networks. J. Syst. Eng. Electron. 2010, 21, 81-87. [CrossRef]

36. Ge, S.S.; Hang, C.C.; Lee, T.H.; Zhang, T. Stable Adaptive Neural Network Control; Springer Science and Business Media: Berlin/Heidelberg, Germany, 2013.

37. Zhu, Q.D.; Liu, Y.C.; Wen, G.X. Adaptive neural network output feedback control for stochastic nonlinear systems with full state constraints. ISA Trans. 2020, 101, 60-68. [CrossRef] [PubMed]

38. Bai, W.W.; Li, T.S.; Tong, S.C. NN reinforcement learning adaptive control for a class of nonstrict-feedback discrete-time systems. EEE Trans. Cybern. 2020, 50, 4573-4584. [CrossRef]

39. Deng, Y.J.; Zhang, X.K; Zhang, G.Q.; Han, X. Adaptive neural tracking control of strict-feedback nonlinear systems with event-triggered state measurement. ISA Trans. 2021. [CrossRef]

40. Arnold, L.; Lefever, R. Stochastic Nonlinear Systems in Physics, Chemistry, and Biology; Spring: Berlin/Heidelberg, Germany, 1981.

41. Liu, Z.G.; Wu, Y.Q. Modelling and adaptive tracking control for flexible joint robots with random noises. Int. J. Control. 2014, 87, 2499-2510. [CrossRef]

42. Diao, S.Z.; Sun, W.; Su, S.F. Neural-based adaptive event-triggered tracking control for flexible-joint robots with random noises. Int. J. Robust Nonlinear Control. 2020. [CrossRef] 